\title{
Phospho-valproic acid inhibits pancreatic cancer growth in mice: Enhanced efficacy by its formulation in poly-(L)-lactic acid-poly(ethylene glycol) nanoparticles
}

\author{
GEORGE MATTHEOLABAKIS ${ }^{1,4}$, RUIXUE WANG ${ }^{2}$, BASIL RIGAS $^{1,3}$ and GERARDO G. MACKENZIE ${ }^{1-3,5}$ \\ Departments of ${ }^{1}$ Medicine and ${ }^{2}$ Family, Population and Preventive Medicine, and ${ }^{3}$ Stony Brook Cancer Center, \\ Stony Brook University, Stony Brook, NY 11794-8175; ${ }^{4}$ Department of Basic Pharmaceutical Sciences, University of \\ Louisiana at Monroe, Monroe, LA 71201; ${ }^{5}$ Department of Nutrition, University of California, Davis, CA 95616-5270, USA
}

Received April 17, 2017; Accepted July 11, 2017

DOI: $10.3892 /$ ijo. 2017.4103

\begin{abstract}
Pancreatic cancer (PC) is one of the most difficult cancers to treat. Since the current chemotherapy is inadequate and various biological approaches have failed, the need for agents that have a potential to treat PC is pressing. Phosphovalproic acid (P-V), a novel anticancer agent, is efficacious in xenograft models of human PC and is apparently safe. In the present study, we evaluated whether formulating P-V in nanoparticles could enhance its anticancer efficacy. In a mouse model of Kras/pancreatitis-associated PC, P-V, orally administered, inhibited the incidence of acinar-to-ductal metaplasia by $60 \%$. To improve its efficacy, we formulated P-V in five different polymeric nanoparticles. Poly-(L)-lactic acidpoly(ethylene glycol) (PLLA-PEG) nanoparticles proved the optimal formulation. PLLA-PEG improved P-V's pharmacokinetics in mice enhancing the levels of P-V in blood. Compared to control, P-V formulated in PLLA-PEG suppressed the growth of MIA PaCa-2 xenografts by $81 \%$, whereas $\mathrm{P}-\mathrm{V}$ alone reduced it by $51 \%(\mathrm{P}<0.01)$. Furthermore, $\mathrm{P}-\mathrm{V}$ formulated in PLLA-PEG inhibited acinar-to-ductal metaplasia in mice with activated Kras, reducing it by $87 \%(\mathrm{P}<0.02)$. In both disease models, P-V suppressed STAT3 phosphorylation at the Ser727 and Tyr705 residues; STAT3 is the pivotal molecular target of P-V. In conclusion, $\mathrm{P}-\mathrm{V}$ is a promising agent against $\mathrm{PC}$, and its formulation in PLLA-PEG nanoparticles enhances its efficacy by improving its pharmacokinetics.
\end{abstract}

\section{Introduction}

Each year approximately 400,000 patients worldwide are diagnosed with pancreatic cancer (PC). Because of its aggressive

Correspondence to: Dr Gerardo G. Mackenzie, Department of Nutrition, University of California, 3135 Meyer Hall, One Shields Avenue, Davis, CA 95616-5270, USA

E-mail: ggmackenzie@ucdavis.edu

Key words: pancreatic cancer, STAT3, phospho-valproic acid, PLLA-PEG nanoparticles, polymeric nanoparticles nature, late diagnosis, rapid disease progression and resistance to chemotherapy, PC is often fatal within 6 months (1). The disappointing performance of current treatments and the magnitude of the clinical problem necessitate the development of new agents for PC.

A useful strategy in designing novel agents is the chemical modification of known drugs in order to optimize their pharmacological properties, primarily their efficacy (2-4). Following this approach, we synthesized phospho-valproic acid (P-V; MDC-1112), a novel valproic acid derivative. We have reported that $\mathrm{P}-\mathrm{V}$ is safe, based on genotoxicity and animal toxicity data, and displays greater efficacy than valproic acid against PC in xenograft models in mice (5). The anticancer effect of $\mathrm{P}-\mathrm{V}$ is mediated to a large extent through the signal transducer and activator of transcription 3 (STAT3) pathway (5). STAT3 plays a significant role in the pathogenesis of PC, being associated with malignant tumor initiation, transformation and progression (6-8). Because it regulates several pathways important in tumorigenesis (9), STAT3 is recognized as a potential drug target for PC $(10,11)$.

Although $\mathrm{P}-\mathrm{V}$ is efficacious against $\mathrm{PC}$, its pharmacological profile limits the extent of its anticancer effect. For this reason, we explored alternatives that would enhance P-V's pharmacokinetic properties. Over the past decade, there has been an increasing interest in using nanotechnology for cancer therapy, in order to enhance drug efficacy and lower drug toxicity. Among these, polymer-based drug delivery systems, including the poly-L-lactic acid (PLLA) polymers, are being developed to improve the diagnosis and treatment of various diseases, including cancer (12). PLLA polymers are of particular interest, since they are biodegradable, biocompatible and FDA-approved (13). A major breakthrough in the nanoparticle field is the use of hydrophilic polymers, for example poly(ethylene glycol) (PEG), to efficiently coat conventional nanoparticle surfaces (14). Amphiphilic copolymers with PEG, such as PLLA-PEG, form a protective hydrophilic and flexible corona around the polymeric core of the nanoparticles that repel plasma proteins, avoid opsonization and exhibit prolonged circulation (14).

In the present study, we assessed whether formulating $\mathrm{P}-\mathrm{V}$ in polymeric nanoparticles could improve the pharmacokinetics 
of P-V and enhance its anticancer effect. We show that P-V formulated in PLLA-PEG has potent anticancer activity towards PC in various mouse models of PC. We also report on its pharmacokinetic properties. Our results further support the anticancer potential of P-V and support the use of PLLA-PEG for its delivery.

\section{Materials and methods}

Materials. The copolymers PCL(10,000)-PEG(5,000), PCL $(5,000)-\operatorname{PEG}(5,000)$ and PCL $(3,600)-\operatorname{PEG}(5,000)$ were obtained from Polymer Source, Inc. (Dorval, QC, Canada). The copolymers PS(2,000)-PEG(2,000) and PLLA(5,000)-PEG(2,000) were obtained from PolySciTech (West Lafayette, IN, USA). Phospho-valproic acid (P-V; MDC-1112) was a gift from Medicon Pharmaceuticals, Inc. (Stony Brook, NY, USA). All general solvents and reagents were of HPLC grade or the highest grade commercially available.

Preparation and characterization of polymeric nanoparticles containing $P-V$. We used the single emulsification and solvent evaporation method to prepare the polymeric nanoparticles from each respective polymer (15). Briefly, a dichloromethane solution of P-V and each polymer (ratio 15:100 w/w respectively) was transferred in an aqueous solution of sodium cholate. The mixture was probe sonicated at $15 \mathrm{~W}$ for $2 \mathrm{~min}$ and the resulting oil/water emulsion was gently stirred at room temperature in a fume hood until complete evaporation of the organic solvent. The nanoparticles were purified by centrifugation and reconstituted in the appropriate volume of water to achieve the desired drug concentration.

The morphology of the nanoparticles was examined using scanning electron microscopy. A drop of the nanoparticle suspension was transferred onto a small metal plate. After drying, the sample was coated with gold using an Edward 150B sputterer, depositing $6 \mathrm{~nm}$ of gold, and transferred in the sample holder of a LEO 1550 SFEG-SEM electron microscope using $2 \mathrm{kV}$ accelerating voltage and with a secondary-electron detector.

The size and $\zeta$ (zeta) potential of the nanoparticles were determined using dynamic light scattering (DLS) and microelectrophoresis, respectively, $10 \mathrm{~min}$ after diluting the samples in phosphate-buffered saline (PBS) and housing them at $25^{\circ} \mathrm{C}$ using a ZetaPlus Brookhaven instrument (Brookhaven Instruments Corp., Holtsville, NY, USA). Entrapped P-V was quantified with high-performance-liquid-chromatography (HPLC) Waters Alliance 2695 equipped with a Waters 2998 photodiode array detector $(220 \mathrm{~nm}$ ) (Waters Corp., Milford, MA, USA) and a Hypersil C18 column (150 x $4.6 \mathrm{~mm}$, particle size $3 \mu \mathrm{m}$; Thermo Fisher Scientific, Waltham, MA, USA). We dissolved a small known amount of lyophilized nanoparticle suspension into $1 \mathrm{ml}$ of acetonitrile to determine the drug loading and encapsulation efficiency for the formulation. The mobile phase followed a gradient between buffer $\mathrm{A}\left(\mathrm{H}_{2} \mathrm{O}\right.$, acetonitrile, trifluoroacetic acid 94.9:5:0.1 v/v/v) and buffer B (acetonitrile).

Cell viability assay. Human PC (Panc-1 and MIA PaCa-2) cell lines, which carry the most common mutations for pancreatic cancer, KRAS and TP53 mutations, as well as they present high (Panc-1) and very high (MIA PaCa-2) constitutive STAT3 activation expression levels $(16,17)$, were purchased from the American Type Culture Collection (ATCC; Manassas, VA, USA), which characterizes them using cytogenetic analysis. We have not authenticated these cell lines. These cells were grown at $37^{\circ} \mathrm{C}$ in $5 \% \mathrm{CO}_{2}$ in Dulbecco's modified Eagle's medium (DMEM) supplemented with $10 \%$ fetal calf serum (FCS), penicillin $(50 \mathrm{U} / \mathrm{ml})$ and streptomycin $(50 \mu \mathrm{g} / \mathrm{ml})$. All the cell lines were characterized for cell morphology and growth rate and passaged in our laboratory less than 6 months after being received.

After treatment with P-V or P-V formulated in PLLA-PEG for 24 or $48 \mathrm{~h}$, cell viability was determined by the reduction of 3-(4,5-dimethylthiazol-2-yl)-2,5-diphenyltetrazolium bromide dye (MTT), following the manufacturer's protocol (Promega, Madison, WI, USA) (4). Briefly, MIA PaCa-2 and Panc-1 cells were plated in 96-well plates (5,000 cells/well), and the next day, cells were treated, in at least triplicates, with varying concentrations of $\mathrm{P}-\mathrm{V}$ or $\mathrm{P}-\mathrm{V}$ formulated in PLLA-PEG, and incubated for 24 or $48 \mathrm{~h}$ with the drugs, at $37^{\circ} \mathrm{C}$ in $5 \% \mathrm{CO}_{2}$. After 24 or $48 \mathrm{~h}$ of incubation, $10 \mu \mathrm{l} /$ well 3-(4,5-dimethylthiazol-2-yl)-2,5-diphenyltetrazolium bromide (MTT 1) (Sigma-Aldrich, St. Louis, MO, USA) was added and incubated at $37^{\circ} \mathrm{C}$ for $4 \mathrm{~h}$. MTT2 stop solution of $10 \%$ sodium dodecyl sulfate (SDS) and $1 \mathrm{M} \mathrm{HCl}$ at $100 \mu \mathrm{l} /$ well was added as solubilizing solution after incubation. Absorbance at $590 \mathrm{~nm}$ (reference at $670 \mathrm{~nm}$ ) using SpectraMax i3 plate reader (Molecular Devices, Sunnyvale, CA, USA) was used to determine cell viability.

Western blot analysis. Whole cell fractions were isolated from tumors as previously described (3). Western blots were performed as previously described $(18,19)$. Briefly, after treatment with the drugs, cells were scraped on ice, washed with ice-cold PBS and lysed in RIPA lysis buffer (Sigma-Aldrich). Protein concentration was determined using the Bradford method (Bio-Rad Laboratories, Hercules, CA, USA). Aliquots of total fractions containing 25-40 $\mu \mathrm{g}$ protein were separated by reducing $10-12.5 \%(\mathrm{w} / \mathrm{v})$ polyacrylamide gel electrophoresis and electroblotted to nitrocellulose membranes. The membranes were probed overnight with antibodies against p-STAT3 ${ }^{\text {Tyr705 }}$ (cat. no. 9145; Cell Signaling Technology, Danvers, MA, USA), p-STAT3 ${ }^{\text {Ser727 }}$ (cat. no. sc-135649; Santa Cruz Biotechnology, Santa Cruz, CA, USA). $\beta$-actin (cat. no. A1978; Sigma-Aldrich) was used as the loading control. After incubation, for $90 \mathrm{~min}$ at room temperature, in the presence of the secondary antibody (HRP-conjugated; 1:5,000 dilution), the conjugates were visualized by chemiluminescence.

Animal studies. All animal experiments were approved by our Institutional Animal Care and Use Committee at Stony Brook University.

Pancreatitis-accelerated carcinogenesis in mice with activated Kras. In a first study, 2-month-old p48-Cre;Kras ${ }^{G 12 D}$ mice in $\mathrm{C} 57 \mathrm{Bl} / 6 \mathrm{~J}$ background (a gift from Dr Howard Crawford) were injected intraperitoneally with saline or cerulein $(50 \mu \mathrm{g} / \mathrm{kg}$ hourly, six times a day, 2 days a week, for 3 weeks; $n=7 /$ group). Cerulein-injected mice were divided in 
vehicle or P-V treatment groups. P-V $(150 \mathrm{mg} / \mathrm{kg})$ was given 5 days a week by oral gavage, starting on the day of the first cerulein injection. On day 21 , mice were euthanized and the pancreas was excised and fixed in formalin and processed for morphological studies.

In a second study, 10-month-old p48-Cre;Kras ${ }^{G 12 D}$ mice were injected intraperitoneally with saline or cerulein (250 $\mu \mathrm{g} / \mathrm{kg}$, once per day for two consecutive days). Ceruleininjected mice were divided in vehicle or PLLA-PEG P-V treatment groups ( $\mathrm{n}=5-6 / \mathrm{group}) . \mathrm{P}-\mathrm{V}(20 \mathrm{mg} / \mathrm{kg}$ ) formulated in PLLA(5k)-PEG(2k) was given intravenously twice a week, starting on the day of the first cerulein injection. P-V formulated in PLLA(5k)-PEG(2k) was administered intravenously to utilize the potential advantages of the enhanced permeability and retention effect observed in solid tumors. On day 21, mice were euthanized and the pancreas was excised and fixed in formalin or snap-frozen for further analysis.

Nude mouse xenograft studies. Female BALB/c nude mice (5-6-weeks old; Charles River Laboratories, Wilmington, MA, USA) were subcutaneously injected with $1.5 \times 10^{6}$ MIA PaCa- 2 cells in $100 \mu 1$ PBS into the right and left flanks. When tumors reached $200 \mathrm{~mm}^{3}$, mice (n=7/group) were randomized into groups receiving 2-hydroxypropyl- $\beta$-cyclodextrins (vehicle control), empty PLLA(5k)-PEG(2k) (PLLA), P-V $20 \mathrm{mg} / \mathrm{kg}$ reconstituted in 2-hydroxypropyl- $\beta$-cyclodextrins $(\mathrm{P}-\mathrm{V})$ or P-V $(20 \mathrm{mg} / \mathrm{kg})$ formulated in PLLA(5k)-PEG(2k) given intravenously twice a week for 21 days. Tumor volume was calculated as [length $\mathrm{x}$ width $\mathrm{x}$ (length + width/2) $\mathrm{x} 0.56$ ], as previously described (5). Animals were sacrificed and tumors were removed, weighed and stored for further analysis.

Histological examination. At necropsy, pancreas was fixed in $10 \%$ phosphate-buffered formalin for $24 \mathrm{~h}$. After dehydration the sample was embedded in paraffin blocks, and four-micrometer-thick sections were processed routinely, stained with H\&E and scored by a pathologist blinded to sample identity. The presence and extent of pancreatic ductal metaplasia were scored based on the degree of epithelial stratification and nuclear atypia according to histopathology criteria, and expressed as the percent of lesion over the total pancreas area, as we have previously shown (20).

Immunohistochemistry. Immunohistochemical staining for PCNA (sc-15402) and p-STAT3 ${ }^{\text {Ser727 }}$ (sc-135649; both from Santa Cruz Biotechnology), $\alpha$-amylase (cat. no. 3796) and p-STAT3 ${ }^{\text {Tyr705 }}$ (cat. no. 9145; both from Cell Signaling Technology) was performed as previously described $(20,21)$. Briefly, paraffin-embedded sections ( $4 \mu \mathrm{m}$ thick) were deparaffinized, rehydrated and microwave-heated for $10 \mathrm{~min}$ in $0.01 \mathrm{~mol} / 1$ citrate buffer $(\mathrm{pH} 6.0)$ for antigen retrieval, and $3 \% \mathrm{H}_{2} \mathrm{O}_{2}$ was applied to block endogenous peroxidase activity. After 15 min of incubation with blocking serum, the primary antibody or control $\operatorname{IgG}$ (dilution 1:50) was applied and incubated overnight at $4^{\circ} \mathrm{C}$. Slides were washed three times with PBS for 5 min each time. The biotinylated secondary antibody and the streptavidin-biotin complex (Invitrogen, Carlsbad, CA, USA) were applied, each for $30 \mathrm{~min}$ at room temperature with an interval washing. After rinsing with PBS, the slides were immersed for $5 \mathrm{~min}$ in the coloring substrate 3,3'-diaminobenzidine (DAB; Sigma-Aldrich), then rinsed with distilled water, counterstained with hematoxylin, dehydrated and coverslipped.

Scoring. At least 5 fields per sample (at magnification, x200) were scored independently by one investigator blinded to the identity of the samples. For quantification of p-STAT3 and PCNA, cells with a blue nucleus were considered unlabeled, while those with a brown nucleus were considered labeled. We calculated the percentage of positive cells by dividing the number of labeled cells by the number of cells in each field and multiplying by 100. Quantitation of amylase positive area was performed on independent, singly DAB stained sections using the ImageJ Immunohistochemistry Image Analysis software (NIH, Bethesda, MD, USA).

Statistical analysis. Results are expressed as mean \pm SEM. Differences between the groups were determined by one-factor analysis of variance followed by the Tukey's test for multiple comparisons. $\mathrm{P}<0.05$ was statistically significant.

\section{Results}

$P-V$ prevents pancreatitis-accelerated carcinogenesis in mice with activated Kras. We have previously shown that $\mathrm{P}-\mathrm{V}$ reduces the growth of subcutaneous and orthotopic human PC xenografts in nude mice (5). We now investigated whether $\mathrm{P}-\mathrm{V}$ could inhibit Kras-driven pancreatic carcinogenesis in the setting of pancreatitis induced by cerulein. For this purpose, we treated 2-month-old $\mathrm{p} 48$-Cre; $\mathrm{Kras}^{G 12 D}$ mice with cerulein (50 $\mu \mathrm{g} / \mathrm{kg}$; 6-hourly injections twice per week for 3 weeks) to induce pancreatitis. Concomitant with pancreatitis induction and continuing for the following 3 weeks, mice were treated with P-V $150 \mathrm{mg} / \mathrm{kg} / \mathrm{days}$ five times a week or vehicle. In p48-Cre; $\mathrm{Kras}^{G 12 D}$ mice, cerulein treatment produced a significant increase in ductal metaplasia lesions in $\mathrm{p} 48$-Cre $; \mathrm{Kras}^{\mathrm{GI2D}}$ mice, significantly replacing the exocrine compartment (Fig. 1). This was accompanied by the minimal expression of amylase, an acinar marker, and enhanced Alcian blue staining, which characterizes PanINs (7). P-V $150 \mathrm{mg} / \mathrm{kg} / \mathrm{day}$ prevented cerulein-induced acinar-to-ductal metaplasia by $60 \%(\mathrm{P}<0.05)$, an effect associated with increased amylase expression, decreased Alcian blue staining areas, and reduced cerulein-stimulated ductal cell proliferation $(\mathrm{P}<0.01$; Fig. 1$)$. Importantly, $\mathrm{P}-\mathrm{V}$ was well tolerated by the mice, showing no body weight loss throughout the treatment.

$P$-V formulated in PLLA(5k)-PEG(2k) nanoparticles increases circulating drug levels. Since the treatment of PC remains challenging, the task of improving therapies relies on the development of additional technologies that overcome the low accessibility of drugs to the pancreas. P-V is rapidly hydrolyzed by carboxyesterases when administered intravenously. We used (2-hydroxypropyl)- $\beta$-cyclodextrin to solubilize the hydrophobic P-V in water and after i.v. injection, the drug was not detectable even 5 min post-injection. To overcome this limitation and improve the pharmacokinetics of P-V, we incorporated the drug in polymeric nanoparticles of various polymers and determined its pharmacokinetics. A panel of five different polymers composed of poly-caprolactone (PCL) at 

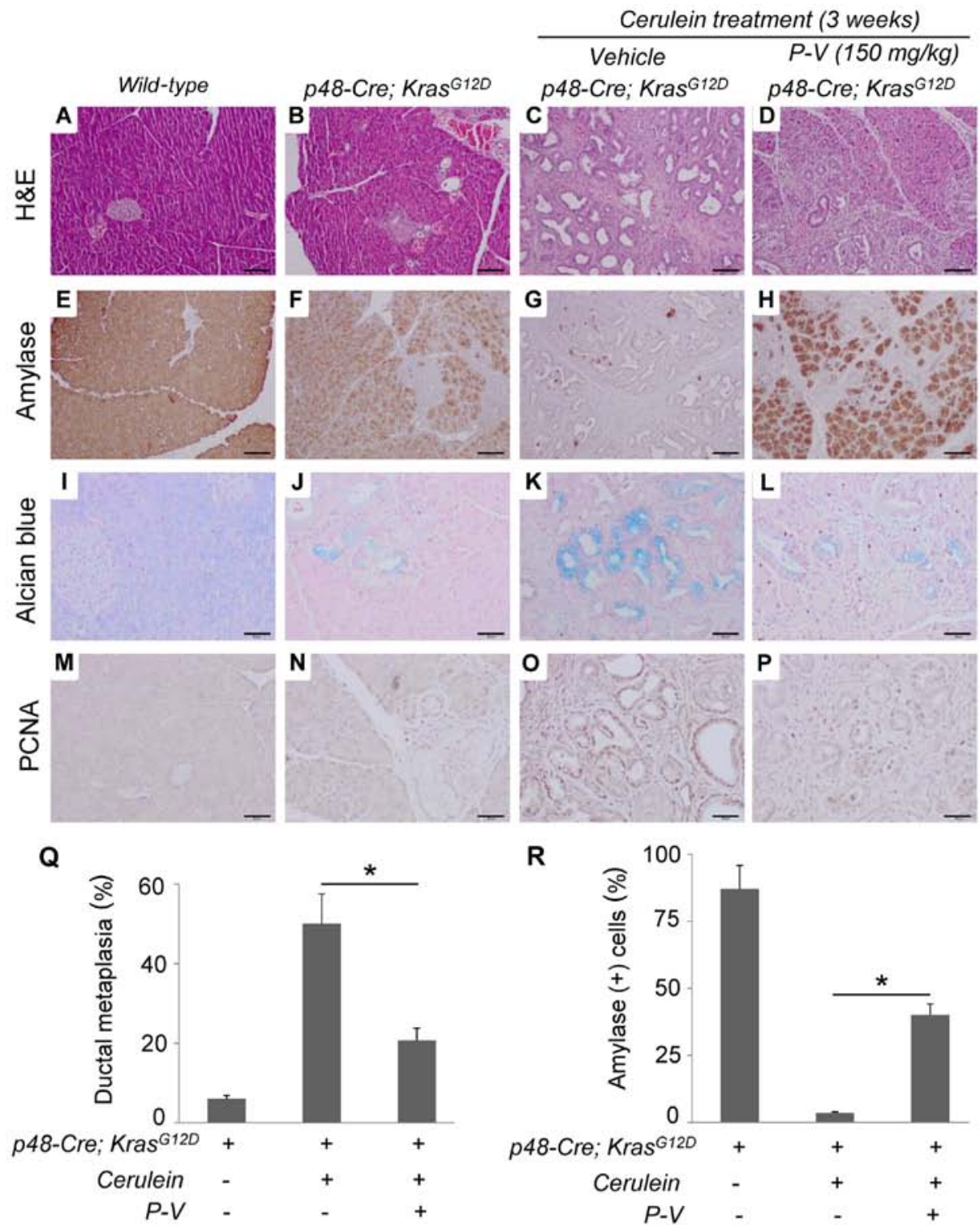

$\mathbf{R}$
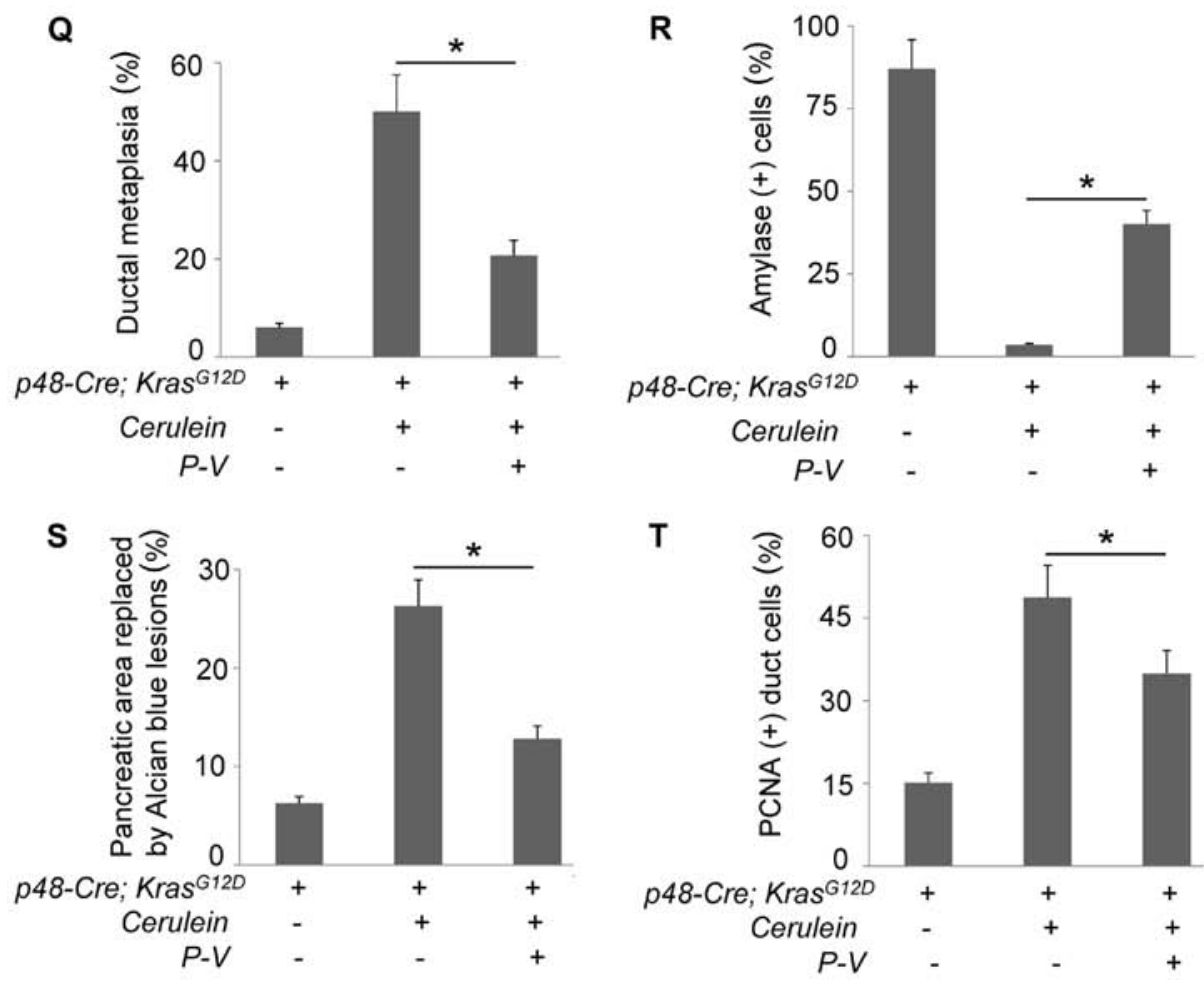

Figure 1. P-V protects against pancreatitis-accelerated carcinogenesis in mice with activated Kras. Two-month-old mice with activated Kras ( $p 48$-Cre;LSL$\operatorname{Kras}^{G 12 D}$ ) were injected with PBS (B, F, J and N), cerulein $(\mathrm{C}, \mathrm{G}, \mathrm{K}$ and $\mathrm{O})$ or cerulein $+\mathrm{P}-\mathrm{V}(\mathrm{D}, \mathrm{H}, \mathrm{L}$ and $\mathrm{P})$ and sacrificed 3 weeks after treatment. Wild-type mice were used as controls (A, E, I and M). The following stains were performed: (A-D) H\&E; representative photographs are shown at x10 magnification. (E-H) Amylase; representative photographs are shown at X10 magnification. (I-L) Alcian blue stain; representative photographs are shown at x20 magnification. (M-P) PCNA; representative photographs are shown at x20 magnification. (Q) The percent of ductal metaplasia per total pancreas was quantified and expressed as the mean $\pm \mathrm{SEM} ;{ }^{*} \mathrm{P}<0.01$. (R) Amylase staining images were quantified and results are expressed as the percentage of amylase positive staining per field; ${ }^{*} \mathrm{P}<0.01$. (S) Alcian blue positive staining in total pancreas area was quantified and results expressed as the percentage of pancreatic area replaced by alcian blue lesions; ${ }^{*} \mathrm{P}<0.01$. (T) PCNA staining images were quantified and results expressed as the percentage of PCNA positive pancreatic duct cells; ${ }^{*} \mathrm{P}<0.01$. Scale bars, $100 \mu \mathrm{m}$ for $\mathrm{H} \& \mathrm{E}$ and amylase; $50 \mu \mathrm{m}$ for Alcian blue and PCNA.

molecular weights of 3.6, 5 and $10 \mathrm{kDa}$, poly-styrene (PS) of $2 \mathrm{kDa}$ or poly-L-lactic acid of $5 \mathrm{kDa}$, all copolymerized with polyethylene glycol of 2 or $5 \mathrm{kDa}$, were tested for improving the P-V pharmacokinetic profile (Table I). All formulations 
Table I. Characterization of polymeric nanoparticles.

\begin{tabular}{lccccr}
\hline & & & & \multicolumn{2}{c}{ P-V blood levels $(\mu \mathrm{M})$} \\
\cline { 5 - 6 } Formulation & Size $(\mathrm{nm})$ & PDI & Z-potential $(\mathrm{mV})$ & 5 min & $1 \mathrm{~h}$ \\
\hline PCL(5k)-PEG(5k) & 81.3 & 0.181 & -6.32 & 0.6 & 0.4 \\
PCL(3.6k)-PEG(5k) & 83 & 0.821 & 0 & 0.0 & 0.0 \\
PS(2k)-PEG(2k) & $\mathrm{N} / \mathrm{A}$ & 1 & $\mathrm{~N} / \mathrm{A}$ & 2.3 & 0.5 \\
PCL(10k)-PEG(5k) & 115.2 & 0.104 & -6.22 & 0.9 & 0.0 \\
PLLA(5k)-PEG(2k) & 81.3 & 0.181 & -14.7 & 41.0 & 20.0
\end{tabular}

A panel of polymers composed of poly-caprolactone (PCL) at molecular weights of 3.6, 5 and $10 \mathrm{kDa}$, poly-styrene (PS) of $2 \mathrm{kDa}$ or polyL-lactic acid of $5 \mathrm{kDa}$, all copolymerized with polyethylene glycol of 2 or $5 \mathrm{kDa}$, were used for improving P-V's PK profile, as described in Materials and methods.
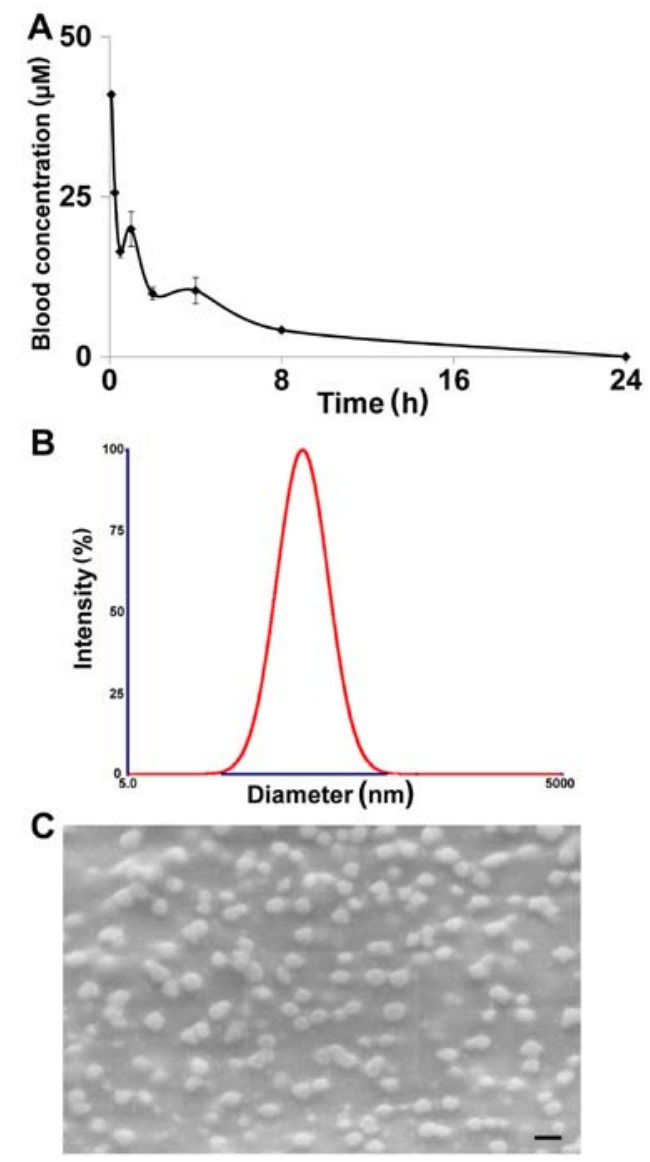

Figure 2. Formulation of P-V in PLLA(5k)-PEG(2k) polymeric nanoparticles increases P-V blood levels. (A) Blood PK for P-V incorporated in PLLA(5k)PEG(2k) nanoparticles in mice ( $\mathrm{n}=2$ per time-point). (B) Hydrodynamic diameter distribution of PLLA(5k)-PEG(2k) polymeric nanoparticles determined using DLS as described in Materials and methods. (C) Scanning electron microscopy image depicting the morphology of the PLLA(5k)PEG(2k) nanoparticles loaded with P-V. Scale bar, $200 \mu \mathrm{m}$.

were prepared by the emulsion-solvent evaporation method, and their hydrodynamic diameter, polydispersity index and zeta potential were determined. Table I summarizes the results.

PLLA(5k)-PEG(2k) nanoparticles generated $\mu \mathrm{M}$ blood levels of P-V (41 and $20 \mu \mathrm{M}$, for $5 \mathrm{~min}$ and $1 \mathrm{~h}$, respectively). The 24-h pharmacokinetic profile of the PLLA(5k)-PEG(2k) nanoparticles demonstrated a prolonged and sustained presence of the drug, which was detectable up to $8 \mathrm{~h}$ after the injection (Fig. 2A). The drug's $\mathrm{AUC}_{0-24 \mathrm{~h}}$ was determined at $117.5 \mu \mathrm{M} / \mathrm{h}$, with $\mathrm{T}_{\max }$ at the first time-point of $5 \mathrm{~min}$ and $\mathrm{C}_{\max }$ of $41 \mu \mathrm{M}$.

The basic characteristics of the PLLA(5k)-PEG(2k) nanoparticles were: a size of $81.3 \mathrm{~nm}$ (Fig. 2B); a polydispersity index of 0.181 and a zeta potential of $-14.7 \mathrm{mV}$. In addition, the nanoparticles were spherical and homogeneous in size as observed under the scanning electron microscope (Fig. 2C). The drug loading for these nanoparticles was $5 \%$ and the encapsulation efficiency was $13.4 \%$.

In contrast, the other tested formulations led to significantly less P-V levels in the blood at $5 \mathrm{~min}$ and $1 \mathrm{~h}$ post intravenous injection, without exceeding the concentration of $3 \mu \mathrm{M}$ in any case (Table I). To note, we were unable to determine the size and zeta potential for the formulation prepared with PCL(3.6k)-PEG(5k) and PS(2k)-PEG(2k). It is conceivable that these polymers resulted in the formation of micelles that their respective size was below the detection limit of our equipment.

Overall, PLLA(5k)-PEG(2k) nanoparticles provided a significantly improved pharmacokinetic profile for P-V compared to the other tested formulations. In addition, PLLA-PEG co-polymers are considered biocompatible and biodegradable, and appear safe based on previous toxicity studies (15). Thus, we chose PLLA(5k)-PEG(2k) nanoparticles as the formulation of choice for $\mathrm{P}-\mathrm{V}$ and we used this formulation in the studies described below.

$P$-V formulated in PLLA(5k)-PEG(2k) nanoparticles reduces $P C$ growth in vitro and in vivo. Initially, we evaluated the cytotoxicity of P-V incorporated in PLLA(5k)-PEG(2k) (PLLA-P-V) in two human PC cell lines, MIA PaCa-2 and Panc- 1 and compared the effect of P-V alone. The values of $24 \mathrm{~h} \mathrm{IC}_{50}$ were as follows: MIA PaCa-2 cells: $\mathrm{P}-\mathrm{V}=73.2 \mu \mathrm{M}$ and PLLA-P-V=67.5 $\mu \mathrm{M}$; Panc-1 cells: $\mathrm{P}-\mathrm{V}=57.7 \mu \mathrm{M}$ and PLLA-P-V=35.3 $\mu \mathrm{M}$ (Fig. 3A). On the other hand, P-V formulated in cyclodextrins led to higher $\mathrm{IC}_{50}$ values in both cell lines. PLLA(5k)-PEG(2k) by itself had no effect on cell growth (data not shown).

We then investigated the effect of PLLA-P-V in MIA PaCa-2 subcutaneous xenografts in nude mice and compared it to that of P-V. When compared to Panc-1 xenografts, MIA PaCa-2 

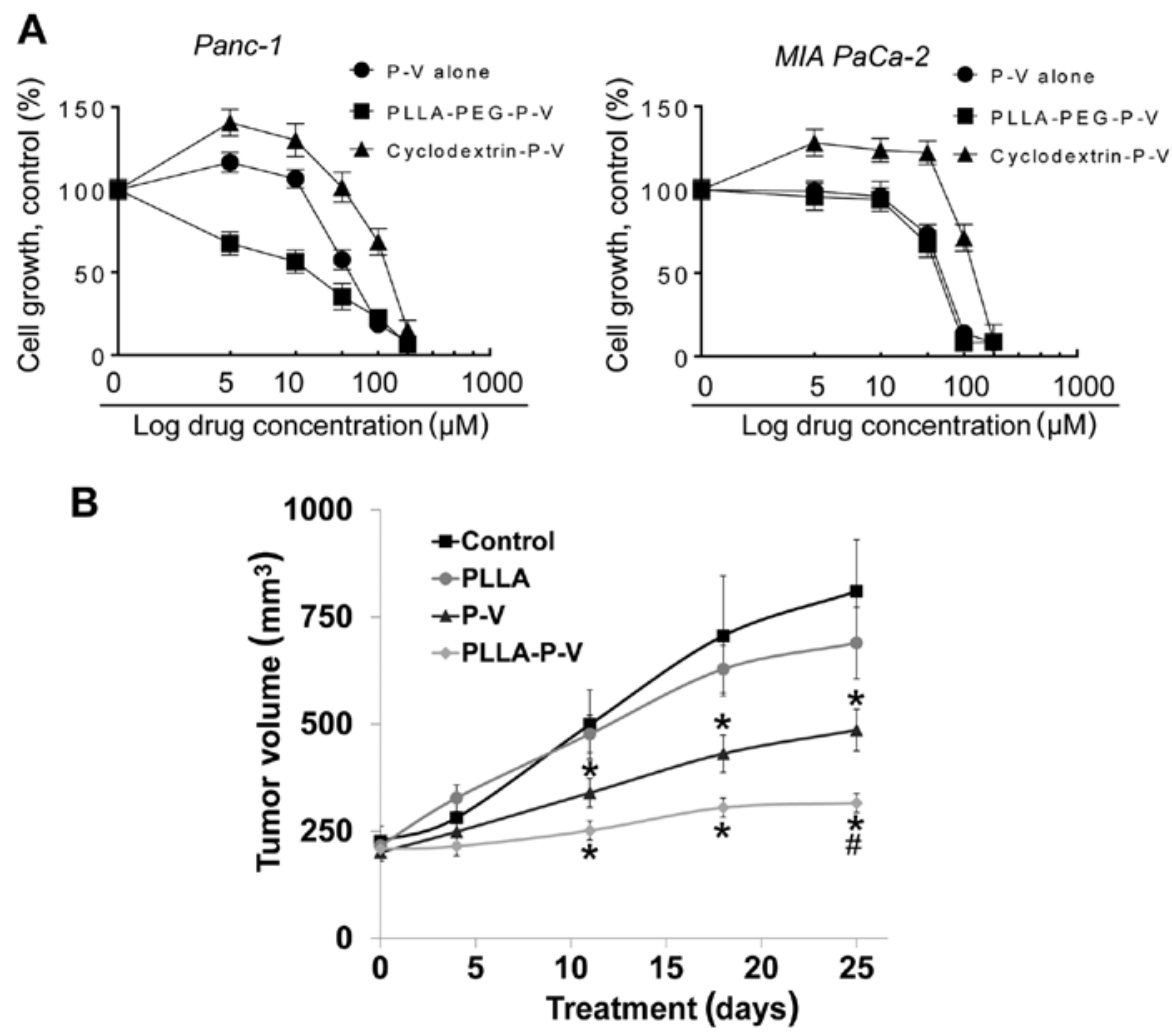

Figure 3. P-V formulated in PLLA(5k)-PEG(2k) polymeric nanoparticles inhibits the growth of human PC in vitro and in xenografts. (A) Cell viability was determined in Panc-1 and MIA PaCa-2 cells after $24 \mathrm{~h}$ of incubation with P-V, P-V formulated in cyclodextrin or P-V formulated in PLLA(5k)-PEG(2k) nanoparticles. Results are expressed as \% control. (B) Nude mice bearing MIA PaCa- 2 xenografts were treated with P-V dissolved in 2 -hydroxypropyl- $\beta$ cyclodextrins (P-V) or P-V formulated in PLLA(5k)-PEG(2k) polymeric nanoparticles (PLLA-P-V), given i.v. at a dose of 20 mg/kg twice a week for 25 days. MIA PaCa-2 tumor volume growth of control, PLLA, P-V and P-V formulated in PLLA(5k)-PEG(2k) (P-V) treated mice. Values, mean \pm SEM. ${ }^{*} \mathrm{P}<0.01$ vs. control group. ${ }^{\text {}} \mathrm{P}<0.05$ vs. $\mathrm{P}-\mathrm{V}$ group.

xenografts present some advantages as they are better grafted and they grow at a higher speed (22-24), better mimicking the aggressiveness of PC. Thus, we explored the efficacy of PLLA-P-V in MIA PaCa-2 xenografts. When tumors reached a volume $200 \mathrm{~mm}^{3}$, mice were randomly divided into four groups: vehicle control, empty PLLA-PEG, P-V dissolved in 2-hydroxypropyl- $\beta$-cyclodextrins (P-V) and PLLA-P-V-treated groups. Mice in the PLLA-P-V group received P-V $(20 \mathrm{mg} / \mathrm{kg})$ formulated in PLLA(5k)-PEG(2k) given intravenously twice a week for 25 days. When compared to controls, the inhibitory effect of PLLA-P-V became statistically significant starting 11 days after the start of the treatment $(\mathrm{P}<0.01)$. Compared to vehicle-treated controls, PLLA-P-V reduced tumor growth by $81 \%(\mathrm{P}<0.01)$, whereas $\mathrm{P}-\mathrm{V}$ dissolved in 2-hydroxypropyl$\beta$-cyclodextrins, given at an equi-dose, reduced it by $51 \%$, indicating a 1.58-fold reduction in the rate of tumor growth for PLLA-P-V when compared to plain P-V (Fig. 3B). Compared to controls, both effects are statistically significant $(\mathrm{P}<0.01)$, as is the difference between the effects of PLLA-P-V and P-V groups $(\mathrm{P}<0.05)$. PLLA-P-V was well tolerated by the mice, which showed no weight loss or other signs of toxicity. Throughout the study the body weight of mice receiving PLLA-P-V, was comparable to that of the control group.

P-V formulated in PLLA(5k)-PEG(2k) nanoparticles reduces pancreatic ductal metaplasia incidence. To gain additional information on P-V formulated in PLLA(5k)-PEG(2k) as a therapeutic agent for PC, we evaluated the efficacy of PLLA-P-V in Kras-driven pancreatic carcinogenesis in the setting of pancreatitis (Fig. 4A). Ten-month-old p48-Cre;Kras ${ }^{G 12 D}$ mice already presented with $100 \%$ penetrance of PanIN lesions, with mice replaced their exocrine compartment by $50 \%$. Treatment with cerulein $(250 \mu \mathrm{g} / \mathrm{kg})$, once a day for 2 consecutive days, led to an acceleration of ductal metaplasia incidence in the 10-month-old p48-Cre;Kras ${ }^{G 12 D}$ mice. This effect was accompanied by enhanced PCNA staining and minimal expression of amylase. PLLA-P-V treatment at $20 \mathrm{mg} / \mathrm{kg}$ twice a week for 3 weeks reduced ductal metaplasia incidence by $87 \%(\mathrm{P}<0.05)$ in transgenic mice, compared to cerulein-treated controls (Fig. 4B). This effect was associated with reduced ductal cell proliferation and increased amylase expression $(\mathrm{P}<0.01$; Fig. 4C-E).

P-V formulated in PLLA(5k)-PEG(2k) nanoparticles inhibits STAT3 signaling in PC. In PC cells in culture and xenografts, $\mathrm{P}-\mathrm{V}$ inhibits STAT3 activation (5) a signaling molecule overexpressed in PC and considered a promising therapeutic target (11). In the present study, we tested whether P-V could inhibit STAT3 activation during Kras-driven metaplasia induced by pancreatitis, evaluating by immunohistochemistry the activation of STAT3 by phosphorylation at two critical sites: Ser727 and Tyr705 (Fig. 5A-C). Cerulein-treated mice had significantly more p-STAT3 $3^{\text {Ser727 }}$ positive duct cells than Kras control mice, while P-V completely blocked it (Fig. 5B). 

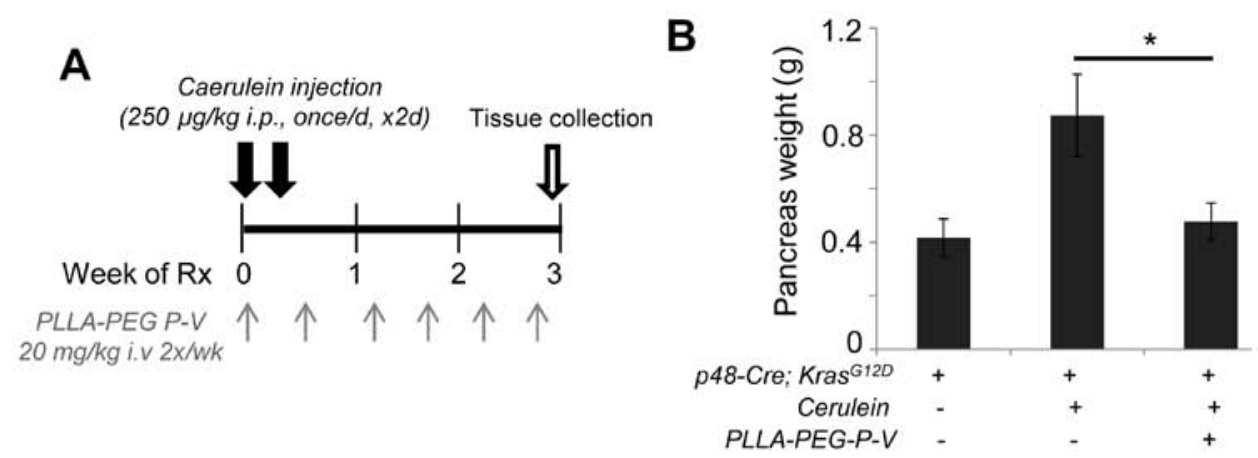

C
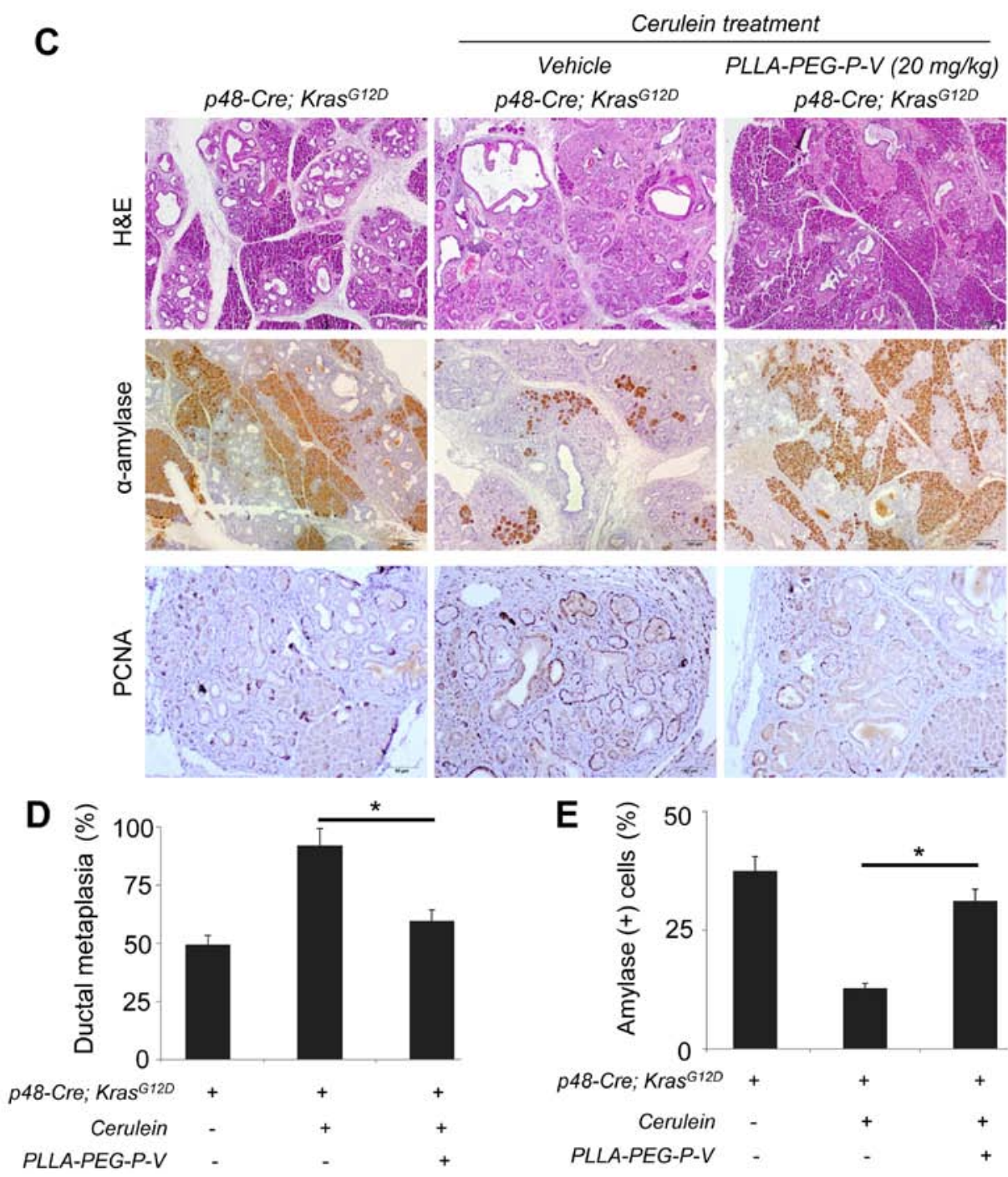

Figure 4. P-V formulated in PLLA(5k)-PEG(2k) polymeric nanoparticles reduces ductal metaplasia formation in mice with activated Kras. (A) Cerulein treatment protocol. Ten-month-old mice with activated Kras $\left(p 48-C r e ; L S L-K r a s^{G 12 D}\right)$ were injected with PBS or cerulein and sacrificed 3 weeks post-injection. At the day of the first injection of cerulein, mice were treated with PLLA(5k)-PEG(2k) polymeric nanoparticles administered i.v. twice a week for 3 weeks as shown. (B) Pancreas weight at sacrifice. Values, mean \pm SEM. " $\mathrm{P}<0.05$ (C). The following stains were performed: H\&E; representative photographs are shown at $\mathrm{x} 4$ magnification. Amylase; representative photographs are shown at $\mathrm{x} 4$ magnification. PCNA; representative photographs are shown at $\mathrm{x} 10$ magnification. (D) The extent of ductal metaplasia lesions was quantified and expressed as percent of ductal metaplasia per total pancreas area. "P<0.05. (E) Amylase staining images were quantified and results expressed as the percentage of amylase positive staining per field; " $\mathrm{P}<0.01$. Scale bars, $200 \mu \mathrm{m}$ for $\mathrm{H} \& \mathrm{E}$ and amylase; $50 \mu \mathrm{m}$ for PCNA.

Moreover, there were significantly more $\mathrm{p}-\mathrm{STAT} 3^{\mathrm{Ty} 705}$ positive stromal cells after cerulein treatment; P-V-treatment decreased this by half $(\mathrm{P}<0.01$; Fig. $5 \mathrm{C})$.

We next evaluated whether P-V formulated in PLLA(5k)PEG(2k) also inhibited STAT3 phosphorylation. As shown in Fig. 5D, PLLA-P-V reduced p-STAT3 ${ }^{\text {Ser727 }}$ and p-STAT3 ${ }^{\text {Tyr705 }}$ levels in MIA PaCa-2 xenografts, compared to control. A reduction in STAT3 ${ }^{\text {Ser727 }}$ phosphorylation levels was also observed by immunohistochemistry. While cerulein-treated mice had significantly more p-STAT3 ${ }^{\text {Ser727 }}$ positive duct cells than Kras control mice, treatment with PLLA-P-V significantly $(\mathrm{P}<0.05)$ reduced $\mathrm{p}-\mathrm{STAT} 3^{\mathrm{Ser} 727}$ levels by $60.4 \%$ (Fig. $5 \mathrm{E}$ ). 
A
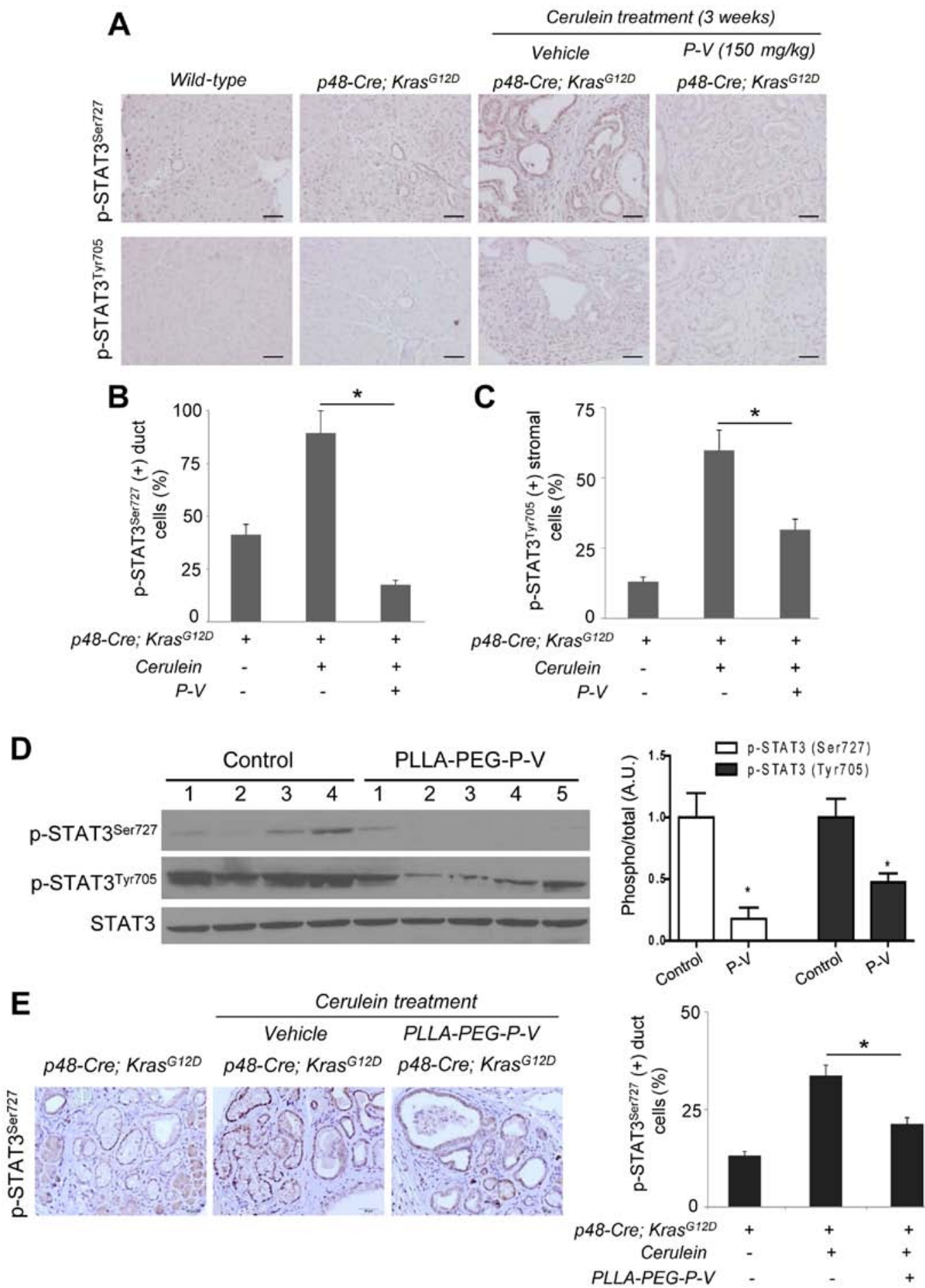

Figure 5. P-V alone and P-V formulated in PLLA(5k)-PEG(2k) polymeric nanoparticles inhibits STAT3 activation in human pancreatic tumor xenografts and in Kras-mice. (A) Mice with activated Kras ( 4 48-Cre;LSL-Kras ${ }^{G 12 D}$ ) were injected with PBS or cerulein or cerulein + P-V-treated, and sacrificed 3 weeks after treatment. Wild-type mice were used as controls. The following stains were performed: p-STAT3 ${ }^{\text {Ser727 }}$; representative photographs are shown at x20 magnification. p-STAT3 ${ }^{\text {Tyr705; }}$ representative photographs are shown at $\mathrm{x} 20$ magnification. (B) Tissue sections, immunostained for p-STAT3 ${ }^{\text {Ser727 }}$, were quantified and results are expressed as the percent of p-STAT3 ${ }^{\text {Ser727 }}$ positive pancreatic duct cells per field; " $\mathrm{P}<0.01$. (C) Tissue sections, immunostained for p-STAT3 ${ }^{\text {Tyr705 }}$, were quantified and results expressed as the percentage of p-STAT3 ${ }^{\text {Tyr705 }}$ positive cells per field; ${ }^{*} \mathrm{P}<0.01$. Scale bars, $50 \mu \mathrm{m}$. (D) Lysates from tumor samples obtained from MIA PaCa-2 xenografts were analyzed for p-STAT3 ${ }^{\text {Tyr705}}$, p-STAT3 ${ }^{\text {Ser727 }}$ and STAT3 proteins by immunoblotting. Each lane represents a different tumor sample. (E) p-STAT3 ${ }^{\text {Ser727}}$; representative photographs are shown at 20x magnification. Tissue sections immunostained for p-STAT3 ${ }^{\text {Ser727 }}$ were quantified and results expressed as the percent of p-STAT3 ${ }^{\text {Ser } 27}$ positive pancreatic duct cells per field; " $\mathrm{P}<0.05$. Scale bars, $50 \mu \mathrm{m}$.

\section{Discussion}

$\mathrm{PC}$ is an almost universally fatal disease, with unsatisfactory treatment options (25). Clearly, this unmet medical need requires developing new agents and novel strategies for PC treatment. We have previously shown that the novel compound $\mathrm{P}-\mathrm{V}$ is a strong inhibitor of PC in xenograft models in mice (5). In the present study, we have extended our work and show that $\mathrm{P}-\mathrm{V}$ is also effective in transgenic Kras-driven models of PC and that the formulation of P-V in PLLA(5k)-PEG(2k) 
nanoparticles, which improves the $\mathrm{P}-\mathrm{V}$ pharmacological profile, results in an attractive alternative for future drug design. The anticancer efficacy of P-V and its apparent lack of toxicity make it a promising candidate drug for the treatment of PC.

Cancer-associated inflammation is a molecular key feature in PC. In response to acute pancreatitis induced by cerulein, acini transiently de-differentiate into duct-like structures that express embryonic factors characteristic of pancreatic embryonic progenitors. However, the presence of mutant Kras compromises the ability of acinar cells to regenerate following acute pancreatitis and locks damaged cells in a persistently de-differentiated, ductal state that can rapidly give rise to PanINs (26). This model of pancreatitis-accelerated carcinogenesis in Kras-mutant mice is an established model to study the potential chemopreventive effect of novel agents. As shown by our findings using this model, $\mathrm{P}-\mathrm{V}$ reduces the incidence of PC by $60 \%$. This effect is similar to the effect we observed for $\mathrm{P}-\mathrm{V}$ in human xenograft models (5).

To enhance the P-V bioavailability and efficacy, we tested various potential copolymers to formulate nanoparticles. PLLA(5k)-PEG(2k) nanoparticles proved to be ideal for $\mathrm{P}-\mathrm{V}$, leading to a significantly improved pharmacokinetic profile with increased levels of intact drug in the blood, when compared to the other tested copolymers and to the drug alone. PLLA(5k)-PEG(2k) nanoparticles have been successfully used to entrap anticancer drugs, achieving improved water solubility, bioavailability and antitumor efficacy via the enhanced permeability and retention effect (27). For instance, PLLA(5k)-PEG(2k) co-polymers loaded with doxorubicin and paclitaxel, accumulated in tumors, released the drug slowly (28), which resulted in tumor regression in mice (29). P-V formulated in PLLA(5k)-PEG(2k) exhibited a protection from esterases, leading to longer circulation time of P-V. This can be explained by the steric hindrance produced by the hydrophilic corona formed by the flexible PEG chains being exposed out and away from the solid hydrophobic polymeric core. It is of interest that the other tested polymers, though copolymers containing PEG, were unable to produce such a favorable result for P-V. We suspect that PLLA created the appropriate hydrophobic environment in the solid polymeric core for P-V to remain entrapped. Furthermore, based on our findings in two different PC mouse models, in which the efficacy was $\geq 80 \%$, formulating P-V in PLLA(5k)-PEG(2k) nanoparticles appears an ideal approach to protect $\mathrm{P}-\mathrm{V}$ and enhance its efficacy.

STAT3 plays an essential role in the initiation and progression of PC $(7,8)$ and in the induction of resistance to apoptosis (11). STAT3 has been shown to cooperate with other inflammatory-related transcription factors, such as NFATc1, in pancreatic development (30), and STAT3 activation by IL-6 transignaling promotes progression of PanIN and development of PC (8). Indeed, STAT3 may serve as a mediator of inflammation-associated processes, such as pancreatitis-driven PanIN development. P-V alone and formulated in PLLA(5k)-PEG(2k) nanoparticles inhibited STAT3 phosphorylation at the Ser727 and Tyr705 residues in both the xenografts as well as in the Kras/pancreatitis-associated PC models.

In summary, the novel compound $\mathrm{P}-\mathrm{V}$ is an effective anticancer agent in preclinical models of $\mathrm{PC}$, acting primarily through STAT3 inhibition. Furthermore, its formulation in
PLLA(5k)-PEG(2k) nanoparticles increases its circulation time and enhances its efficacy. Our data further indicate P-V as a promising candidate drug for $\mathrm{PC}$ and suggest its formulation in PLLA(5k)-PEG(2k) nanoparticles for its administration.

\section{Acknowledgements}

The present study was funded in part by grants from NIHCA175699, NIHCA181727 to G.G.M and the Knapp Foundation to B.R.

\section{References}

1. Siegel RL, Miller KD and Jemal A; V1: Cancer statistics, 2016. CA Cancer J Clin 66: 7-30, 2016.

2. Huang L, Mackenzie GG, Sun Y, Ouyang N, Xie G, Vrankova K, Komninou D and Rigas B: Chemotherapeutic properties of phospho-nonsteroidal anti-inflammatory drugs, a new class of anticancer compounds. Cancer Res 71: 7617-7627, 2011.

3. Mackenzie GG, Bartels LE, Xie G, Papayannis I, Alston N, Vrankova K, Ouyang N and Rigas B: A novel Ras inhibitor (MDC-1016) reduces human pancreatic tumor growth in mice. Neoplasia 15: 1184-1195, 2013.

4. Mackenzie GG, Sun Y, Huang L, Xie G, Ouyang N, Gupta RC, Johnson F, Komninou D, Kopelovich L and Rigas B: Phospho-sulindac (OXT-328), a novel sulindac derivative, is safe and effective in colon cancer prevention in mice. Gastroenterology 139: 1320-1332, 2010.

5. Mackenzie GG, Huang L, Alston N, Ouyang N, Vrankova K, Mattheolabakis G, Constantinides PP and Rigas B: Targeting mitochondrial STAT3 with the novel phospho-valproic acid (MDC-1112) inhibits pancreatic cancer growth in mice. PLoS One 8: e61532, 2013.

6. Corcoran RB, Contino G, Deshpande V, Tzatsos A, Conrad C, Benes CH, Levy DE, Settleman J, Engelman JA and Bardeesy N: STAT3 plays a critical role in KRAS-induced pancreatic tumorigenesis. Cancer Res 71: 5020-5029, 2011.

7. Fukuda A, Wang SC, Morris JP IV, Folias AE, Liou A, Kim GE, Akira S, Boucher KM, Firpo MA, Mulvihill SJ, et al: Stat 3 and MMP7 contribute to pancreatic ductal adenocarcinoma initiation and progression. Cancer Cell 19: 441-455, 2011.

8. Lesina M, Kurkowski MU, Ludes K, Rose-John S, Treiber M, Klöppel G, Yoshimura A, Reindl W, Sipos B, Akira S, et al: Stat $3 /$ Socs 3 activation by IL- 6 transsignaling promotes progression of pancreatic intraepithelial neoplasia and development of pancreatic cancer. Cancer Cell 19: 456-469, 2011.

9. Scholz A, Heinze S, Detjen KM, Peters M, Welzel M, Hauff P, Schirner M, Wiedenmann B and Rosewicz S: Activated signal transducer and activator of transcription 3 (STAT3) supports the malignant phenotype of human pancreatic cancer. Gastroenterology 125: 891-905, 2003.

10. Buettner R, Mora LB and Jove R: Activated STAT signaling in human tumors provides novel molecular targets for therapeutic intervention. Clin Cancer Res 8: 945-954, 2002.

11. Sahu RP and Srivastava SK: The role of STAT-3 in the induction of apoptosis in pancreatic cancer cells by benzyl isothiocyanate. J Natl Cancer Inst 101: 176-193, 2009.

12. Mattheolabakis G, Rigas B and Constantinides PP: Nanodelivery strategies in cancer chemotherapy: Biological rationale and pharmaceutical perspectives. Nanomedicine (Lond) 7: 1577-1590, 2012.

13. Soppimath KS, Aminabhavi TM, Kulkarni AR and Rudzinski WE: Biodegradable polymeric nanoparticles as drug delivery devices. J Control Release 70: 1-20, 2001.

14. Brigger I, Dubernet C and Couvreur P: Nanoparticles in cancer therapy and diagnosis. Adv Drug Deliv Rev 54: 631-651, 2002.

15. Mattheolabakis G, Taoufik E, Haralambous S, Roberts ML and Avgoustakis K: In vivo investigation of tolerance and antitumor activity of cisplatin-loaded PLGA-mPEG nanoparticles. Eur J Pharm Biopharm 71: 190-195, 2009.

16. Wei D, Le X, Zheng L, Wang L, Frey JA, Gao AC, Peng Z, Huang S, Xiong HQ, Abbruzzese JL, et al: Stat 3 activation regulates the expression of vascular endothelial growth factor and human pancreatic cancer angiogenesis and metastasis. Oncogene 22: 319-329, 2003. 
17. Deer EL, González-Hernández J, Coursen JD, Shea JE, Ngatia J, Scaife CL, Firpo MA and Mulvihill SJ: Phenotype and genotype of pancreatic cancer cell lines. Pancreas 39: 425-435, 2010

18. Sun Y and Rigas B: The thioredoxin system mediates redoxinduced cell death in human colon cancer cells: Implications for the mechanism of action of anticancer agents. Cancer Res 68: 8269-8277, 2008.

19. Mackenzie GG, Queisser N, Wolfson ML, Fraga CG, Adamo AM and Oteiza PI: Curcumin induces cell-arrest and apoptosis in association with the inhibition of constitutively active NF-kappaB and STAT3 pathways in Hodgkin's lymphoma cells. Int J Cancer 123: 56-65, 2008.

20. Mattheolabakis G, Papayannis I, Yang J, Vaeth BM, Wang R, Bandovic J, Ouyang N, Rigas B and Mackenzie GG: Phosphoaspirin (MDC-22) prevents pancreatic carcinogenesis in mice. Cancer Prev Res 9: 624-634, 2016.

21. Mackenzie GG, Ouyang N, Xie G, Vrankova K, Huang L, Sun Y, Komninou D, Kopelovich L and Rigas B: Phospho-sulindac (OXT-328) combined with difluoromethylornithine prevents colon cancer in mice. Cancer Prev Res (Phila) 4: 1052-1060, 2011.

22. Fogar P, Greco E, Basso D, Habeler W, Navaglia F, Zambon CF, Tormen D, Gallo N, Cecchetto A, Plebani M, et al: Suicide gene therapy with HSV-TK in pancreatic cancer has no effect in vivo in a mouse model. Eur J Surg Oncol 29: 721-730, 2003.

23. Freeman JW, Mattingly CA and Strodel WE: Increased tumorigenicity in the human pancreatic cell line MIA PaCa-2 is associated with an aberrant regulation of an IGF-1 autocrine loop and lack of expression of the TGF-beta type RII receptor. J Cell Physiol 165: 155-163, 1995
24. Mattheolabakis G, Papayannis I, Yang J, Vaeth BM, Wang R, Bandovic J, Ouyang N, Rigas B and Mackenzie GG: Phosphoaspirin (MDC-22) prevents pancreatic carcinogenesis in mice. Cancer Prev Res (Phila) 9: 624-634, 2016.

25. Li J and Saif MW: Advancements in the management of pancreatic cancer. JOP 10: 109-117, 2009.

26. Morris JP IV, Wang SC and Hebrok M: KRAS, Hedgehog, Wnt and the twisted developmental biology of pancreatic ductal adenocarcinoma. Nat Rev Cancer 10: 683-695, 2010.

27. Zhan C, Gu B, Xie C, Li J, Liu Y and Lu W: Cyclic RGD conjugated poly(ethylene glycol)-co-poly(lactic acid) micelle enhances paclitaxel anti-glioblastoma effect. J Control Release 143: 136-142, 2010.

28. Ahmed F, Pakunlu RI, Srinivas G, Brannan A, Bates F, Klein ML, Minko T and Discher DE: Shrinkage of a rapidly growing tumor by drug-loaded polymersomes: $\mathrm{pH}$-triggered release through copolymer degradation. Mol Pharm 3: 340-350, 2006.

29. Kim SC, Kim DW, Shim YH, Bang JS, Oh HS, Wan Kim S and Seo MH: In vivo evaluation of polymeric micellar paclitaxel formulation: toxicity and efficacy. J Control Release 72: 191-202, 2001.

30. Baumgart S, Chen NM, Siveke JT, König A, Zhang JS, Singh SK, Wolf E, Bartkuhn M, Esposito I, Heßmann E, et al: Inflammation-induced NFATc1-STAT3 transcription complex promotes pancreatic cancer initiation by $\mathrm{Kras}^{G 12 D}$. Cancer Discov 4: 688-701, 2014. 